\title{
Orbital-ordering-induced anomalous softening of the ferromagnetic spin waves in perovskite manganites
}

\author{
R. Kajimoto ${ }^{a, *, \dagger}$, H. Yoshizawa ${ }^{a}$, H. Kawano-Furukawa ${ }^{b, \dagger}$, \\ H. Kuwahara ${ }^{\mathrm{c}, \ddagger}$, Y. Tomioka ${ }^{\mathrm{c}}$, and Y. Tokura ${ }^{\mathrm{c}}$ \\ ${ }^{a}$ Neutron Scattering Laboratory, I. S. S. P., Univ. of Tokyo, Tokai, Ibaraki, 319-1106, Japan \\ ${ }^{\mathrm{b}}$ Institute of Physical and Chemical Research, Hirosawa 2-1, Wako, Saitama, 351-0198, Japan \\ ${ }^{\mathrm{c}}$ Joint Research Center for Atom Technology, Tsukuba, Ibaraki 305-8562, Japan
}

(November 16, 2018)

\begin{abstract}
Spin wave excitations were measured in the ferromagnetic phase of $\mathrm{Nd}_{1 / 2} \mathrm{Sr}_{1 / 2} \mathrm{MnO}_{3}$ by neutron scattering. This compound is located in proximity to the A-type antiferromagnetic state, and it shows a clear anisotropy and anomalous softening of the spin wave excitations. The softening in the ferromagnetic phase is induced by the orbital ordering.
\end{abstract}

Keywords: Magnetoresistance - transition metals; Spin waves; Neutron scattering

Recent experimental and theoretical studies revealed that the ordering of the $\mathrm{Mn} e_{g}$ orbitals plays a crucial role to determine physical properties in the perovskite manganites in addition to the well-known double exchange interactions. Especially, the strong influence of the orbital ordering on the magnetism and the transport properties in the antiferromagnetic (AFM) state are widely recognized. For example, the metallic A-type AFM state, in which ferromagnetic (FM) planes stack antiferromagnetically, was suggested to be attributed to the ordering of $d\left(x^{2}-y^{2}\right)$ orbitals of Mn ions in the FM planes. The anisotropic interactions of the $d\left(x^{2}-y^{2}\right)$ orbitals introduce a noticeable two dimensional character in the spin fluctuations 国.

On the other hand, the influence of the orbital ordering in the FM or paramagnetic (PM) state is not clear yet. Accordingly, a neutron scattering studies on the FM and $\mathrm{PM}$ phase of $\mathrm{Pr}_{1 / 2} \mathrm{Sr}_{1 / 2} \mathrm{MnO}_{3}$ and $\mathrm{Nd}_{1 / 2} \mathrm{Sr}_{1 / 2} \mathrm{MnO}_{3}$, which are located in proximity to the A-type AFM state, were performed in order to examine the influence of the orbital ordering on the spin fluctuations. The samples are single crystals, and the measurements were performed on the $(h, l, h)$ scattering plane (in the Pnma setting). Because the space of this paper is limited, we will only present the results for $\mathrm{Nd}_{1 / 2} \mathrm{Sr}_{1 / 2} \mathrm{MnO}_{3}$. Similar results

\footnotetext{
${ }^{*}$ Corresponding author. Fax: +81 35978 5325; e-mail: kaji@phys.ocha.ac.jp

${ }^{\dagger}$ Present address: Faculty of Science, Ochanomizu University, Bunkyo-ku, Tokyo 112-8610, Japan.

${ }_{\ddagger}$ Present address: Faculty of Science and Technology, Sophia University, Chiyoda-ku, Tokyo 102-8554, Japan.
}

were obtained for $\operatorname{Pr}_{1 / 2} \mathrm{Sr}_{1 / 2} \mathrm{MnO}_{3}$, which will be described elsewhere.

$\mathrm{Nd}_{1 / 2} \mathrm{Sr}_{1 / 2} \mathrm{MnO}_{3}$ shows the FM transitions at $T_{\mathrm{C}}=250$ $\mathrm{K}$. With decreasing temperature, the magnetic structure is switched to the CE-type AFM structure at $T_{\mathrm{N}}^{\mathrm{CE}}=160$ $\mathrm{K}$ as a first order transition [2]. In addition, we found that the canted A-type AFM structure appears as a second order transition in the FM phase below $T_{\mathrm{N}}^{\mathrm{A}}=200 \mathrm{~K}$. Its AFM propagation vector is $(0,1,0)$ in reciprocal lattice unit. In this paper, we will denote the direction within or between the FM planes as intraplane or interplane direction, respectively.

Figure 1 (a) shows the spin wave dispersion curves which were measured from the zone center to the zone boundary in the FM phase. Two interesting features were observed. First, the spin wave excitations show strongly anisotropic behavior, which is similar to the A-type AFM phase 11. Secondly, the dispersion curves exhibit severe softening near the zone boundary. Especially, the softening of the dispersion curve along the interplane direction is so strong that the dispersion resembles that of the A-type AFM state. We think these results suggest the existence of the influence of the $d\left(x^{2}-y^{2}\right)$-type orbital ordering in the FM phase. In the following, we shall discuss relations between softening of the interplane spin waves and the orbital ordering in detail. The discussion on the anisotropy of the spin waves has been reported elsewhere [3].

In order to parametrize the softening phenomenogically, we have fitted the observed dispersions to the Heisenberg 

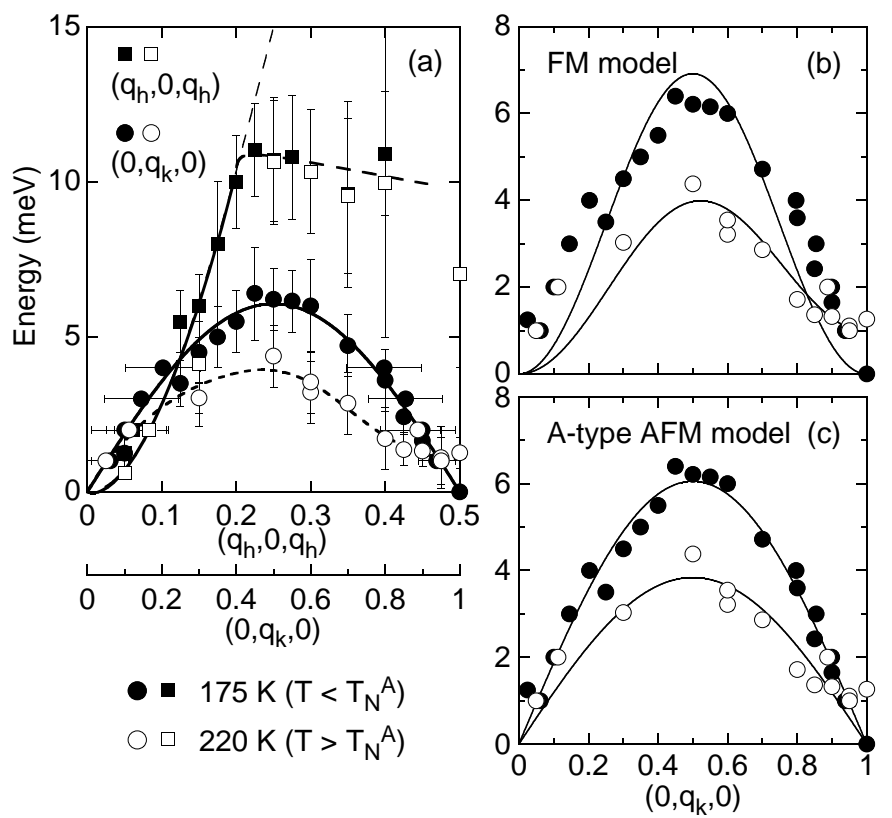

Fig. 1. (a) Spin wave dispersion curves for $\mathrm{Nd}_{1 / 2} \mathrm{Sr}_{1 / 2} \mathrm{MnO}_{3}$ in the FM phase. Squares and circles indicate data measured along the intraplane direction and those along the interplane direction, respectively. Solid symbols are data for $175 \mathrm{~K}$ and open symbols are those for $220 \mathrm{~K}$. (b), (c) Fits to the interplane dispersions based on two models (described in the text).

model for ferromagnets, although the localized spin model is too naive. It is necessary to take the second nearest neighbor exchanges along the interplane direction into account to reproduce the softening of the interplane dispersion. Therefore, we fitted the data by the following equation:

$$
\hbar \omega\left(q_{k}\right)=8 J_{1} S \sin ^{2}\left(\frac{1}{2} \pi q_{k}\right)+8 J_{2} S \sin ^{2}\left(\pi q_{k}\right)
$$

where $J_{1}$ and $J_{2}$ are the exchange integrals for the nearest neighbors and those for the second nearest neighbors along the interplane direction. The results are shown in Fig. 1 (b). Alternatively, we have fitted the data to the Heisenberg model for antiferromagnet, because the interplane dispersions resemble the one for the A-type antiferromagnet. Here, we adopted only the exchanges between the first nearest neighbors. Then, the equation for the dispersion becomes

$$
\hbar \omega\left(q_{k}\right)=-8 J_{1} S\left|\frac{1}{2} \sin \left(\pi q_{k}\right)\right|,
$$

where $J_{1}<0$. The results of the fitting by Eq. (2) is indicated in Fig. 1 (c).

Although Eq. (11) can reproduce the softening, it fails to describe the data for the small $q$ region, and $J_{1}$ becomes very small compared to $J_{2}: 8 J_{1} S=1.0 \mathrm{meV}$ and $8 J_{2} S=$ $3.5 \mathrm{meV}$ for $220 \mathrm{~K}$, while $8 J_{1} S=0 \mathrm{meV}$ and $8 J_{2} S=6.9$
meV for $175 \mathrm{~K}$. In contrast, the observed data are rather well described by the AFM dispersion (Eq. 2) except for high- $q$ region at $220 \mathrm{~K}$. In this case, however, $J_{1}<0$ $\left(8 J_{1} S=-7.7 \mathrm{meV}\right.$ for $220 \mathrm{~K}$ and $-12 \mathrm{meV}$ for $\left.175 \mathrm{~K}\right)$ in spite of the fact that the long range $\mathrm{FM}$ ordering is formed.

The exchange parameters obtained by these procedures are clearly anomalous, and it should be attributed to the inadequacy of the localized Heisenberg spin model. It is clear that theory which takes into account the relevant microscopic mechanism has to be developed. We think the fluctuations of the exchange energy induced by the $d\left(x^{2}-y^{2}\right)$-type orbital correlation may be responsible for the softening of the interplane dispersion. This scenario may also explain the temperature dependence of the inter-

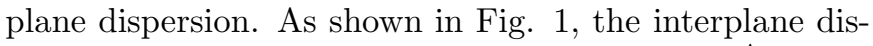
persion has a gap at the zone boundary above $T_{\mathrm{N}}^{\mathrm{A}}$, which reflects that the orbital ordering is not fully stabilized yet. With decreasing temperature, the orbital ordering becomes stable. As a result, the energy gap decreases and eventually vanishes in the canted A-type AFM phase, resulting in a complete A-type AFM dispersion as shown in Fig. 1 (c). The transition to the A-type AFM states may be regarded as a transition by the softening of magnons, on the analogy of the structural transition by the softening of phonons. We would like to note that the similar softening of the FM spin wave dispersion and its temperature dependence was reproduced by the recent theoretical calculations which adopted the orbital correlations [4, 5].

In summary, we found an anomalous softening of the magnon dispersions in the FM phase of $\mathrm{Nd}_{1 / 2} \mathrm{Sr}_{1 / 2} \mathrm{MnO}_{3}$. The softening is attributed to the influence of the orbital ordering in the FM phase, and the FM to A-type AFM transition is induced by the softening of the magnon.

This work was supported by a Grant-In-Aid for Scientific Research from the Ministry of Education, Science and Culture, Japan and by the New Energy and Industrial Technology Development Organization (NEDO) of Japan.

\section{References}

[1] H. Yoshizawa et al., Phys. Rev. B 58, R571 (1998).

[2] H. Kawano et al., Phys. Rev. Lett. 78, 4253 (1997); Physica B 241-243, 289 (1998)

[3] H. Kawano et al., cond-mat/9808286.

[4] S. Ishihara et al., Phys. Rev. B 56, 686 (1997).

[5] G. Khaliullin and R. Kilian, Phys. Rev. B 61, 3494 (2000). 